\title{
MULTIOBJECTIVE GLOBAL OPTIMIZATION OF MECHANICAL SYSTEMS WITH CRACKS ${ }^{1}$
}

\author{
Witold Beluch, Adam DŁugosz \\ Silesian University of Technology, Faculty of Mechanical Engineering, Gliwice, Poland \\ e-mail: witold.beluch@polsl.pl; adam.dlugosz@polsl.pl
}

\begin{abstract}
The paper is devoted to the multiobjective shape optimization of cracked structures. The two main goals are: reduction of the negative crack influence of identified cracks and optimal design of structural elements to reduce the risk of crack occurrence and growth. NURBS (Non-Uniform Rational B-Splines) curves are used to model the structure boundaries. Global optimization methods in the form of evolutionary algorithms are employed. As different optimization criteria are considered simultaneously, the efficient multiobjective optimization method are applied. An in-house multiobjective evolutionary algorithm is proposed as an efficient optimization tool. The dual boundary element method is used to solve the boundaryvalue problem.
\end{abstract}

Keywords: multiobjective optimization, crack, dual BEM, NURBS, Pareto front

\section{Introduction}

Cracks can occur in different structures and affect their strength and lifetime. They may be a result of a technological process or they may arise during operation of a structure.

Stress intensity factors (SIFs) play a crucial role in the description of singular stress fields and the prediction of crack propagation for linear elastic fracture mechanics. The SIF can be defined as (Anderson, 2003)

$$
K=\sqrt{2 E \gamma}
$$

where: $E$ - Young's modulus, $\gamma$ - surface energy density of the material.

SIFs are usually calculated for three linearly independent cracking modes: I - opening mode, II - in-plane shear mode and III - out-of-plane shear mode. Values of SIF can be found in the literature for simple geometries and loading conditions (Tada et al., 2000). In general, SIFs are determined using numerical methods. For this purpose, the J-integral technique (Sun and Jin, 2012) or Williams expansion series (Caicedo and Portela, 2015) can be applied.

The formation of a crack can significantly reduce lifetime of the structure. Continuum damage mechanics (CDM) allows one to describe material deterioration before initiation of the cracks (or voids) based on the continuous variable (Upadhyaya and Sridhara, 2011). In the fracture mechanics approach applied in the present paper, initiation and growth of cracks is treated as a discontinuous phenomenon (Gross and Seelig, 2011). The most common cause of fracture is fatigue crack growth in which the crack grows from a very small to critical size, resulting in damage of the structure with no visible effect. The crack growth rate determining the service life of the structure can be represented in a general form as (Neimitz, 1998)

$$
\frac{d l}{d N}=f(\sigma, l, C, Y, R, \chi)
$$

\footnotetext{
${ }^{1}$ The work is related to a paper at PCM-CMM-2019.
} 
where: $N$ - number of loading cycles, $l$ - current crack length, $\sigma$ - stress expressed by stress amplitude, $C$ - material constants, $Y$ - geometrical parameters of the element or crack, $R=\sigma_{\max } / \sigma_{\min }-$ cycle ratio, $\chi$ - function (functional) representing loading history.

The Paris law allows calculating $f(\cdot)$ function for the velocity of crack propagation between $10^{-9}$ and $10^{-6} \mathrm{~m} /$ cycle

$$
\frac{d l}{d N}=c(\Delta K)^{m}
$$

where: $\Delta K=K_{\max }-K_{\min }, K$ - stress intensity factor for single-mode fracture analysis, $c, m$ - material constants determined experimentally.

There are several criteria for the crack propagation direction (Aliabadi, 2003). The maximum principal stress criterion is based on the postulate that the local crack extension direction $\theta_{t}$ is determined by the condition that the local shear stress is equal to zero

$$
K_{I} \sin \theta_{t}+K_{I I}\left(3 \cos \theta_{t}-1\right)=0
$$

where: $\theta_{t}$ - angular coordinate of the tangent to the crack path, $K_{I}, K_{I I}$ - mode I and II SIFs.

Crack influence on the structure may be reduced by shape modification (optimization) of the structure. There exist two main attitudes to the shape optimization of cracked structures: (i) minimization of the stress intensity factors; (ii) maximization of the fatigue lifetime of the structure. Both aspects are considered in the present paper.

In many real optimization problems, more than one criterion has to be considered. If such criteria are contradictory, a multiobjective optimization task must be formulated.

A multiobjective optimization (MOO) problem is a search for the vector $\mathbf{s}=\left[s_{1}, s_{2}, \ldots, s_{n}\right]^{\mathrm{T}}$, $\mathbf{s} \in \mathbf{D}$, where $\mathbf{D}$ is a set of admissible solutions, simultaneously minimizing the vector of $k$ objective functions (Deb, 2001)

$$
f(\mathbf{s})=\left[f_{1}(\mathbf{s}), f_{2}(\mathbf{s}), \ldots, f_{k}(\mathbf{s})\right]^{\mathrm{T}}
$$

The vector $\mathbf{s}$ has to satisfy $m$ inequality constrains $g_{i}(\mathbf{s}) \geqslant 0, i=1,2, \ldots, m$ and $p$ equality constrains $h_{i}(\mathbf{s})=0, i=1,2, \ldots, p$.

There exist three groups of multiobjective optimization methods (Zitzler et al., 2004): (i) a priori methods, (ii) interactive methods and (iii) a posteriori methods. Groups (i) and (ii) lead to one solution of the optimization. While a priori methods transform multiobjective optimization task into a single-objective one (e.g. weighted sum method or $\varepsilon$-constraint method), interactive methods require interaction with the decision-maker during the optimization for additional information.

The third group of multiobjective optimization methods results in a set of compromise solutions, which provides additional information for decision-maker, necessary to choose one (or more) compromise solution. In the present paper, the Pareto concept of non-dominated solutions (Ehrgott, 2005) is employed. Considering the minimization task, a solution $\mathbf{s}$ is:

- strongly dominated by a solution $\mathbf{s}^{*}$ if

$$
\forall i \in\{1,2, \ldots, k\}: \quad f_{i}\left(\mathbf{s}^{*}\right)<f_{i}(\mathbf{s})
$$

— weakly dominated by the solution $\mathbf{s}^{*}$ if

$$
\forall i \in\{1,2, \ldots, k\}: \quad f_{i}\left(\mathbf{s}^{*}\right) \leqslant f_{i}(\mathbf{s}) \wedge \exists j \in\{1,2, \ldots, k\}: \quad f_{j}\left(\mathbf{s}^{*}\right)<f_{j}(\mathbf{s})
$$

The solution $\mathbf{s}^{*}$ is called Pareto optimal if it is not dominated by any other solution. The set of Pareto optimal outcomes $f\left(\mathbf{s}^{*}\right)$ is called the Pareto front.

Global, usually biologically inspired optimization methods are very popular due to two main reasons: they reduce the risk to find a local solution instead of the global one, and there is no 
necessity to calculate an objective function(s) gradient or Hessian (Talbi, 2009). Moreover, as they process a set (population) of potential solutions, they may be adopted to multiobjective optimization tasks where the set of solutions in the form of Pareto front is sought.

Several papers have been devoted to reduction of negative influence of cracks on mechanical structures. Perera et al. (2009, 2014) focused on application of different metaheuristic multiobjective optimization techniques (evolutionary algorithms, particle swarm optimizer) in damage localization. Shim and Suh (2010) used an evolutionary algorithm (single and multiobjective) for crack identification in beams. Baptista et al. (2015) applied a direct multisearch algorithm to optimize cruciform specimens under fatigue loading. Most of the research focuses on tasks related to identification or localization of cracks in mechanical systems, while the present paper deals with reducing the risk of occurrence or propagation of cracks by optimizing the structure shape. In addition, the above-mentioned problems were solved using the finite element method (FEM), while in the present paper the boundary element method (BEM) is used. It should be noted that BEM allows calculation of the stress field much more accurately than FEA with the same degree of discernment (Brebbia and Walker, 2016). The use of BEM instead of FEM also allows for significant reduction in the calculation time required for remeshing in the case of crack growth. This is crucial for optimization tasks where a single boundary-value problem must be calculated hundreds or thousands times.

\section{The dual boundary element method}

In order to perform the shape optimization procedure, the boundary-value problem must be solved for each shape generated by the optimization algorithm. The BEM is one of the numerical techniques which allows solving boundary-value problems (Brebbia and Dominiquez, 1989). In contrast to the commonly used FEM, it usually does not require discretization of the entire body volume but only its boundary which substantially reduces the size of the problem under consideration. Additionally, BEM gives higher than FEM accuracy of stress calculation for the same characteristic dimensions of the elements.

Application of the boundary element method to linear elastic problems in domains containing no degenerated geometries is straightforward. The displacement $\mathbf{u}(\mathbf{x})$ of an arbitrary point $\mathbf{x}$ can be represented by the boundary displacement integral equation

$$
c(\mathbf{x}) \mathbf{u}(\mathbf{x})=\int_{\Gamma} \mathbf{U}(\mathbf{x}, \mathbf{y}) \mathbf{p}(\mathbf{y}) d \Gamma(\mathbf{y})-\int_{\Gamma} \mathbf{P}(\mathbf{x}, \mathbf{y}) \mathbf{u}(\mathbf{y}) d \Gamma(\mathbf{y}) \quad \mathbf{x} \in \Gamma
$$

where: $\mathbf{U}(\mathbf{x}, \mathbf{y}), \mathbf{P}(\mathbf{x}, \mathbf{y})$ - fundamental solutions of elastostatics, $c(\mathbf{x})$ - constant depending on the collocation point $\mathbf{x}$ position, $\mathbf{y}$ - boundary point, $\mathbf{p}(\mathbf{y})$ - traction vector pertinent to the boundary point $\mathbf{y}, \mathbf{u}(\mathbf{y})$ - displacement vector pertinent to the boundary point $\mathbf{y}, \Gamma$ - boundary.

Degenerated geometries have a form of internal or edge cracks across which the problem field (e.g. displacements) is discontinuous. Direct application of BEM is impossible in such a case as two identical equations (2.1) are applied at both crack surfaces, which leads to a singular set of algebraic equations obtained after discretization of the body.

To overcome this problem different techniques have been proposed in the literature. For linear 2D cracks, Green's functions method may be applied (Snyder and Cruse, 1975). In the multi-region method, the body is divided into parts in the place of crack(s) (Blandford et al., 1981). This method requires higher discretization and needs remeshing if crack propagation is considered. The dual BEM (dBEM) is a technique in which this problem is solved by means of an additional traction boundary equation (Portela et al., 1992)

$$
\frac{1}{2} \mathbf{p}(\mathbf{x})=\mathbf{n}\left(\int_{\Gamma} \mathbf{D}(\mathbf{x}, \mathbf{y}) \mathbf{p}(\mathbf{y}) d \Gamma(\mathbf{y})-\int_{\Gamma} \mathbf{S}(\mathbf{x}, \mathbf{y}) \mathbf{u}(\mathbf{y}) d \Gamma(\mathbf{y})\right) \quad \mathbf{x} \in \Gamma
$$


where: $\mathbf{D}(\mathbf{x}, \mathbf{y}), \mathbf{S}(\mathbf{x}, \mathbf{y})$ - third-order fundamental solution tensors, $\mathbf{n}$ - unit outward normal vector at the collocation point $\mathbf{x}$.

Equation (2.2) is applied on one surface of each crack while equation (2.1) is applied on the opposite side of each crack and on the remaining boundary. Moreover, special boundary elements are used due to singularity of the stress field. Discontinuous boundary elements are used to model crack surfaces while semi-discontinuous boundary elements are used to model the parts of the boundary that are in contact with edge cracks.

\section{MOOPTIM algorithm}

A MOOPTIM (MultiObjective OPTIMization tool) algorithm (Długosz, 2013) makes use of the Pareto concept to find a set of compromise solutions. It is inspired by the well-known evolutionary multiobjective optimization algorithm NSGA-II (Deb et al., 2002). Both algorithms use non-dominated sorting procedures to classify individuals (chromosomes), supported by a crowding mechanism (to preserve diversity of the individuals). The main differences relate to the selection procedure and a different number and types of evolutionary operators.

Two crossover operators: (simple crossover arithmetical crossover) and two mutation operators (uniform mutation and Gaussian mutation) are employed. The selection in MOOPTIM is based on two elements: a non-domination level and a crowding coefficient. As in NSGA-II algorithm, each individual in a population is assigned to one of the fronts according to the non-domination level.

The crowding coefficient is calculated for each individual as an absolute normalized difference of the objective functions values of two neighbouring solutions on a particular front. Exceptions are individuals from the ends of fronts, which are assigned values corresponding to infinite distance. The flowchart of MOOPTIM is presented in Fig. 1.

MOOPTIM has been tested on different multiobjective benchmark problems, like SCH, ZDT1, ZDT2, ZDT3, ZDT4, ZDT6, CONSTR, SRN, TNK (Deb, 1999) showing its superiority in most cases, especially for problems with strong multimodality, non-convex or discontinuous Pareto front.

An example of comparing the effectiveness of MOOPTIM and NSGAII for the ZDT4 problem (with many local Pareto fronts) is shown in Fig. 2. Comparison has been performed for 30 independent runs and for the same number of individuals and the same fitness evaluations for both algorithms. The remaining parameters were set according to suggestions of the algorithms authors. It can be observed that MOOPTIM found a set of Pareto optimal solutions for most runs while NSGA-II often gets stuck in local optima. A detailed comparison of MOOPTIM and NSGA-II can be found in (Długosz, 2013).

The effectiveness of MOOPTIM has also been tested for real problems, e.g. for multiobjective optimization of porous microstructures in two-scale thermoelastic problems (Długosz, 2014) for multiobjective and multiscale optimization of composites (Beluch and Długosz, 2016) and for multiobjective optimization in selected coupled problems (Burczyński and Długosz, 2012).

\section{Formulation of the problem}

The aim of the paper is to perform shape optimization of mechanical structures in order to:

(i) reduce negative crack influence of identified internal stationary crack(s) (Burczyński and Beluch, 2001);

(ii) properly design the structural element to reduce the risk of crack occurrence and growth - for edge cracks (Beluch, 2005). 


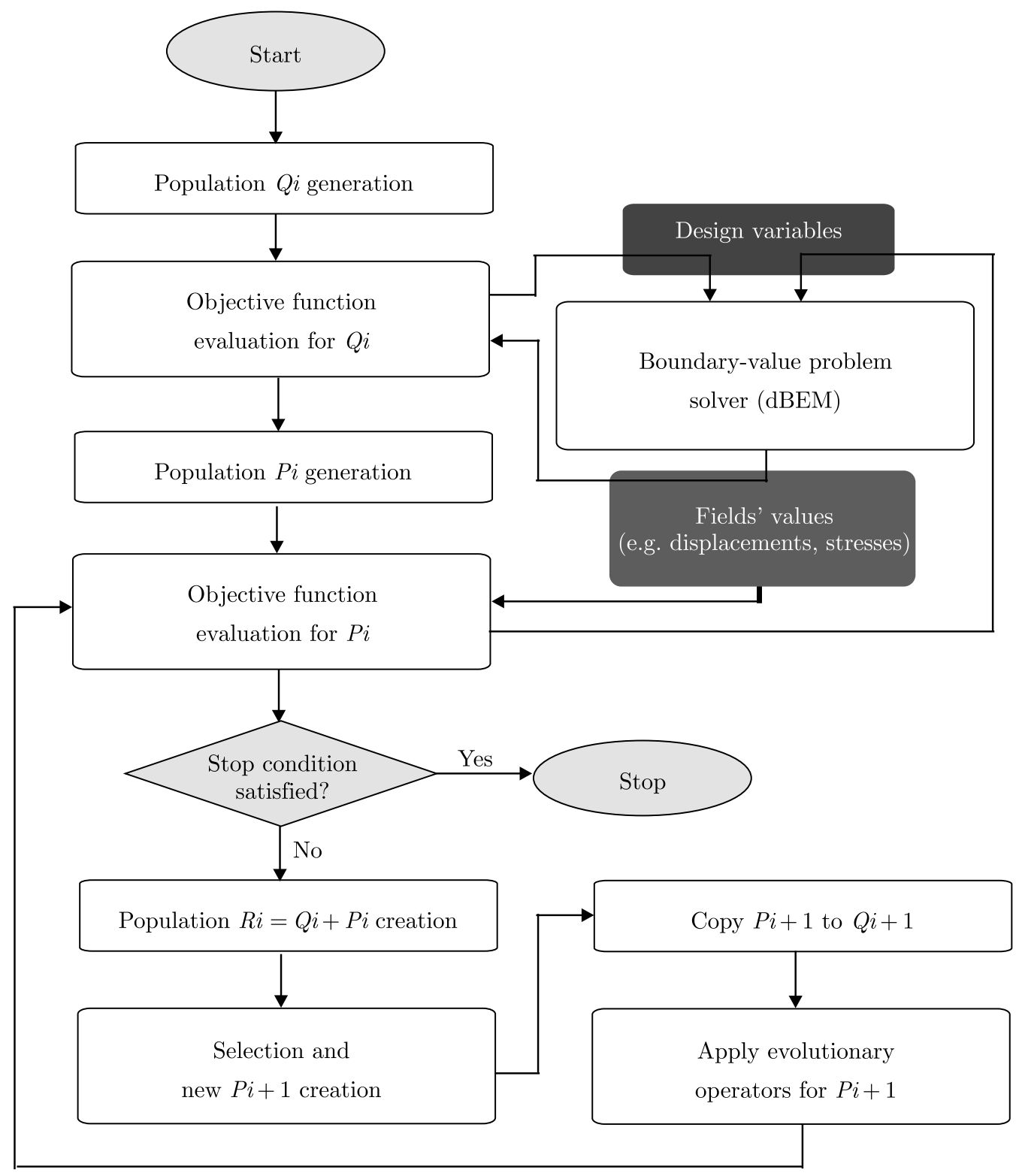

Fig. 1. Flowchart of the MOOPTIM algorithm
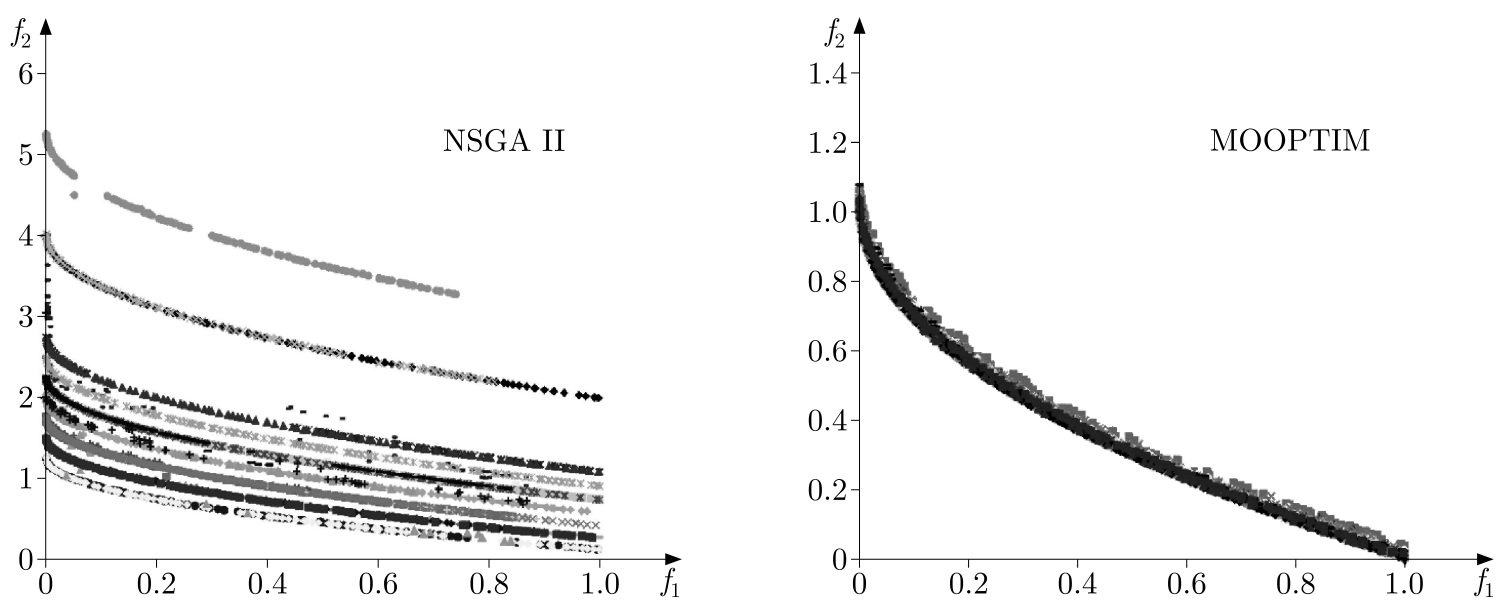

Fig. 2. MOOPTIM-NSGAII comparison on ZDT-4 problem 
The following optimization criteria are considered:

C1: Minimization of the structure volume $V$

$$
\arg \min \{V(\mathbf{s}) ; \mathbf{s} \in \mathbf{D}\}
$$

C2: Minimization of the maximum value of the reduced (von Mises) stress $\sigma_{e q}$

$$
\arg \min \left\{\max \left(\sigma_{e q}(\mathbf{s})\right) ; \mathbf{s} \in \mathbf{D}\right\}
$$

C3: Minimization of the equivalent stress intensity factor (for single stationary, internal crack)

$$
\arg \min \left\{K_{e q}(\mathbf{s})=\sum_{i=1}^{2} \sum_{j=1}^{2} w_{j}^{i}\left|K_{j}^{i}\right| ; w_{j}^{i}=\frac{\left|K_{j}^{i}\right|}{\sum_{i=1}^{2} \sum_{j=1}^{2}\left|K_{j}^{i}\right|} ; \mathbf{s} \in \mathbf{D}\right\}
$$

where: $i$ - crack tip number, $j$ - cracking mode (1 or 2$)$;

C4: Maximization of the number of cycles $N$ necessary to extend the edge crack by length $l$ :

$$
\arg \max \{N(\mathbf{s}) ; \mathbf{s} \in \mathbf{D}\}
$$

As more than one criterion is considered simultaneously, multiobjective optimization is performed.

Shape optimization can result in a large number of design variables of the optimization procedure. To reduce the number of them, the NURBS (Non-Uniform Rational B-Splines) curves are used to model the modified part of the structure boundary. NURBS curves are generalized non-rational B-splines and non-rational and rational Bezier curves. A NURBS curve is defined as (Piegl and Tiller, 1995)

$$
\mathbf{C}(t)=\frac{\sum_{j=0}^{r} N_{j, n}(t) w_{j} \mathbf{P}_{j}}{\sum_{k=0}^{r} N_{k, n}(t) w_{k}} \quad a \leqslant t \leqslant b
$$

where: $\mathbf{P}_{j}$ - control points, $w_{j}$ - weight of control points, $N_{j, n}-n$-degree B-spline basis functions.

An important feature of NURBS curves is the local approximation property: if the control point $\mathbf{P}_{j}$ is moved and/or the weight $w_{j}$ is changed, only a part of the curve on the interval $t \in\left[t_{i}, t_{i+p+1}\right]$ is modified.

Two cases are considered:

I. Modification of the existing structure with the identified internal crack. The aim is to reduce the equivalent stress intensity factor value by modification of the material distribution and/or by adding some material (limitation for the structure volume is introduced).

II. Proper design of the structure to reduce the risk of crack initialization and growth. In this case, dBEM is used twice to calculate the objective function values of a particular individual. First, for each generated possible solution (chromosome representing modified geometry), the boundary-value problem is solved by means of dBEM, and the boundary point with the maximum value of the von Mises stresses is found. Next, the initial crack of assumed length is introduced in the direction perpendicular to the maximum principal stress direction. Finally, the number of cycles $N$ necessary to extend the crack by the assumed length and other values necessary to calculate the objective functions are found by means of dBEM.

\section{Numerical examples}

The shape optimization of 2D structural elements made of an isotropic material in plane stress is performed. The material parameters are Young's modulus $E=200 \mathrm{GPa}$, Poisson's ratio $\nu=0.3$.

According to the results presented in (Długosz, 2013), the parameters of MOOPTIM have been set for both examples as: 
- probability of Gaussian mutation $p_{g m}=0.7$;

- probability of uniform mutation $p_{u m}=0.1$;

- probability of simple crossover $p_{s c}=0.1$;

- probability of arithmetic crossover $p_{a c}=0.1$.

The number of chromosomes $n_{c h}$, the number of genes in each chromosome $n_{g}$ and the number of generations $n_{g}$ vary for different examples.

The design vectors (chromosomes) consist of genes representing coordinates of the NURBS control points.

\subsection{Numerical example 1: case I - two-objective optimization}

The shape optimization of the 2D Y-shaped structure with an identified internal crack is performed (Fig. 3a). The dimensions of the structure are $0.14 \times 0.14 \mathrm{~m}$, the traction values are $T_{1}=T_{2}=10 \mathrm{MPa}$. The boundary of the structure is discretized by 84 boundary elements (Fig. 3b).

The aim of shape optimization is to decrease the influence of the crack on the structure by an appropriate distribution of the material by modification of the free part of the external boundary of the structure. The optimization criteria C1 and C3 are considered simultaneously.

(a)

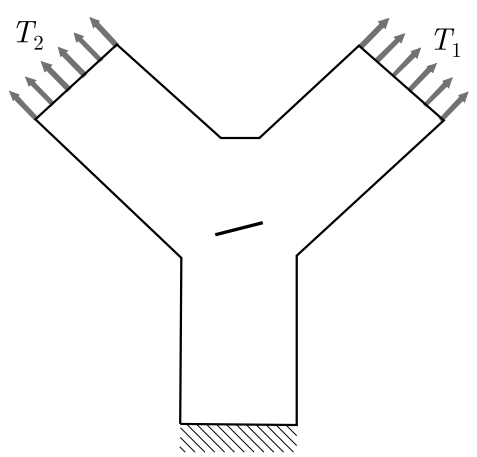

(b)

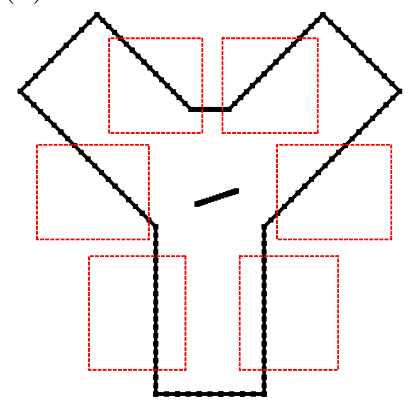

Fig. 3. Structural element 1: (a) shape and boundary conditions, (b) boundary elements and NURBS control points ranges

The values of the structure volume $V_{0}$ and the equivalent SIR $K_{e q 0}$ have been calculated for the initial shape by means of dBEM and they were used to create the following limitations in the optimization procedure: structure volume $V<1.3 V_{0}, V_{0}=8.42461 \mathrm{e}-3 \mathrm{~m}^{2}$, equivalent SIF $K_{e q} \leqslant K_{e q 0}, K_{e q 0}=1.42103$, where the index 0 indicates the initial shape. Six control points coordinates of 3 NURBS curves state 12 design variables (genes). The control points ranges are presented in Fig. 3b.

The remaining parameters of MOOPTIM are the number of chromosomes in population $n_{c h}=50$ and the number of generations (termination condition) $n_{g}=100$.

Several numerical tests have been performed. The best results of the optimization in the form of 2D Pareto fronts for different generations of MOOPTIM and exemplary results for points $A$, $B$ and $C$ are presented in Fig. 4 and collected in Table 1. The figures in Table 1 present optimal shapes (black lines) with the original shape in the background (grey lines).

Obtained shapes are proposals concerning possible ways of "repairing" the structure with identified $\operatorname{crack}(\mathrm{s})$ according to designer (decision-maker) priorities. It can be observed that the Pareto front moves toward the utopia point in consecutive generations, maintaining a high span of it and well-distributed solutions. In the area where the Pareto front is almost flat (C3 > 1.15), it was not possible to evenly fill the front with solutions (which is typical for such cases). The shape of the Pareto front provides additional information on the problem for the designer. In 


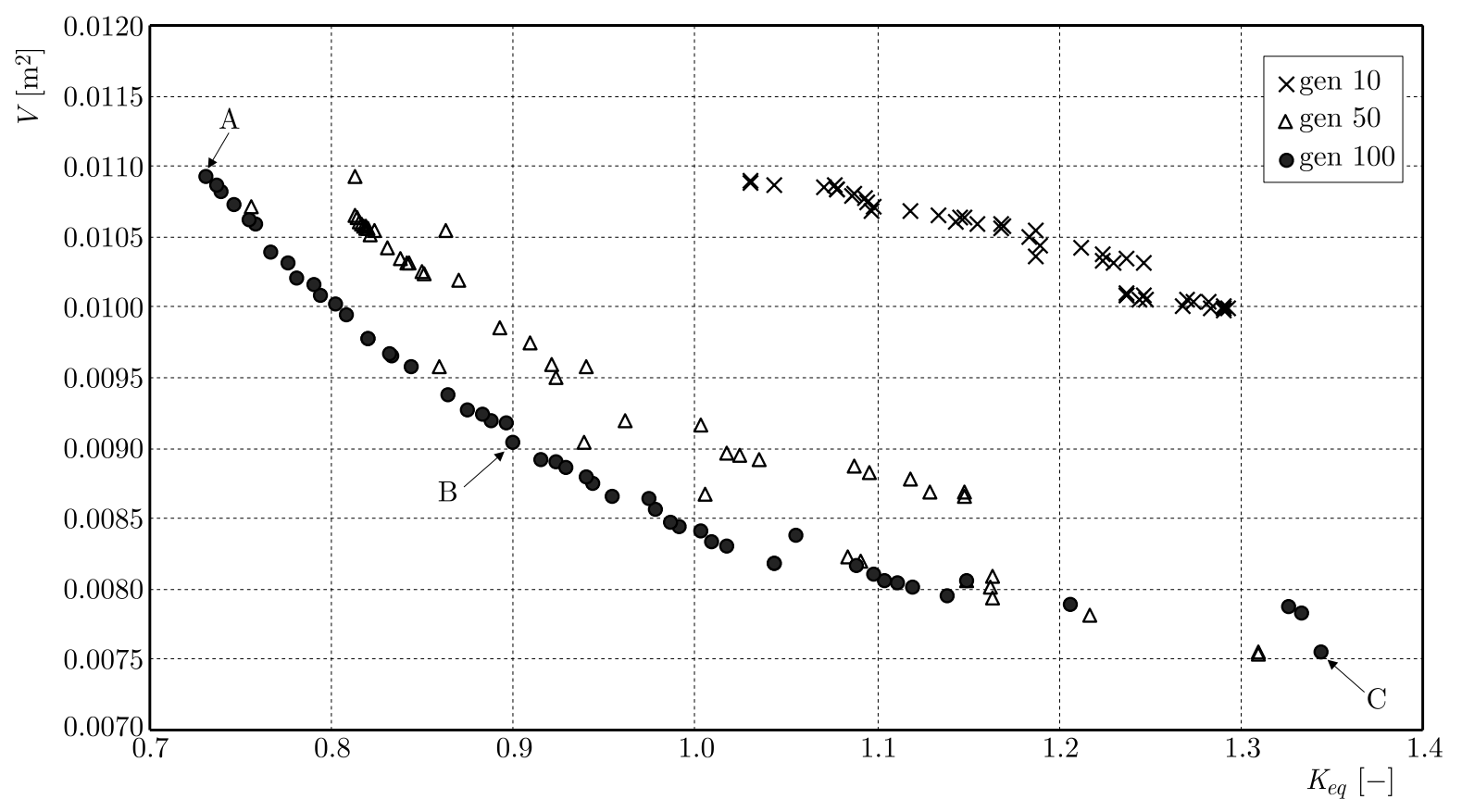

Fig. 4. Pareto front for 2-objective optimization for generations: 10, 50 and 100

Table 1. Optimization results - example 1

\begin{tabular}{|c|c|c|}
\hline & Point $A$ & Point $B$ \\
\hline$K_{e q}[-]$ & 0.731239 &
\end{tabular}

this case, for such a flat region, a small change in the value of criterion $\mathrm{C} 1$ results in rapid changes in criterion $\mathrm{C} 3$.

\subsection{Numerical example 2: case II - three-objective optimization}

The shape optimization of the $2 \mathrm{D}$ cross-shaped structure of external dimensions $0.20 \times 0.20 \mathrm{~m}$ and the initial (crack-free) shape as presented in Fig. 5a is performed. The structure is supported and loaded by 3 tractions: $T_{1}\left(T_{1 x}=2 \mathrm{MPa}, T_{1 y}=2 \mathrm{MPa}\right), T_{2}\left(T_{2 x}=0 \mathrm{MPa}, T_{2 y}=10 \mathrm{MPa}\right)$ and $T_{3}\left(T_{3 x}=-5 \mathrm{MPa}, T_{3 y}=5 \mathrm{MPa}\right)$. The boundary of the structure is discretized by 140 boundary elements.

The aim of shape optimization is minimization of the risk of crack occurrence and growth by modification of parts of the external boundary (represented by thicker, grey lines) as shown in Fig. 5b. The following limitations have been introduced in the optimization procedure: structure volume $V \leqslant 1.1 V_{0}, V_{0}=7.08 \mathrm{e}-02 \mathrm{~m}^{2}$ and the equivalent von Mises stress $\sigma_{e q} \leqslant 200 \mathrm{MPa}$. The modified part of the boundary is modelled by 4 NURBS curves with 3 control points each. 

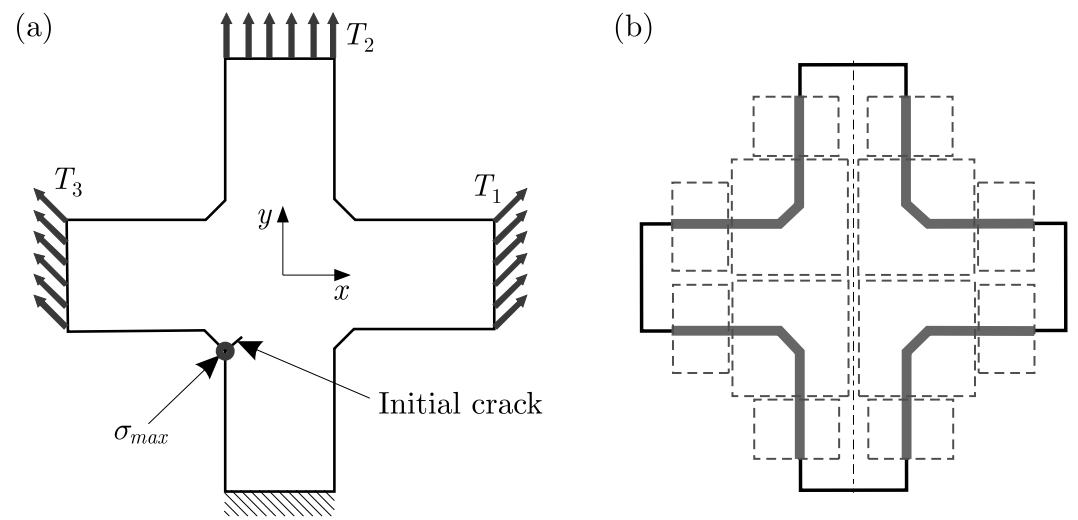

Fig. 5. Structural element 2: (a) shape, boundary conditions and exemplary initial crack, (b) modified parts of the boundary and NURBS control points ranges

As symmetry of the structure about the vertical axis is assumed, the number of design variables is 12 . The optimization criteria $\mathrm{C} 1, \mathrm{C} 2$ and $\mathrm{C} 4$ are considered simultaneously. The parameters of the Paris law are $R=2 / 3, c=4.62 \cdot 10^{-12}, m=3.4$. The location of the maximum value of the equivalent von Mises stress and an exemplary initial crack for the initial shape of the structure are presented in Fig. 6a.

The remaining parameters of MOOPTIM are the number of chromosomes in population $n_{c h}=150$ and the number of generations (termination condition) $n_{g}=300$.

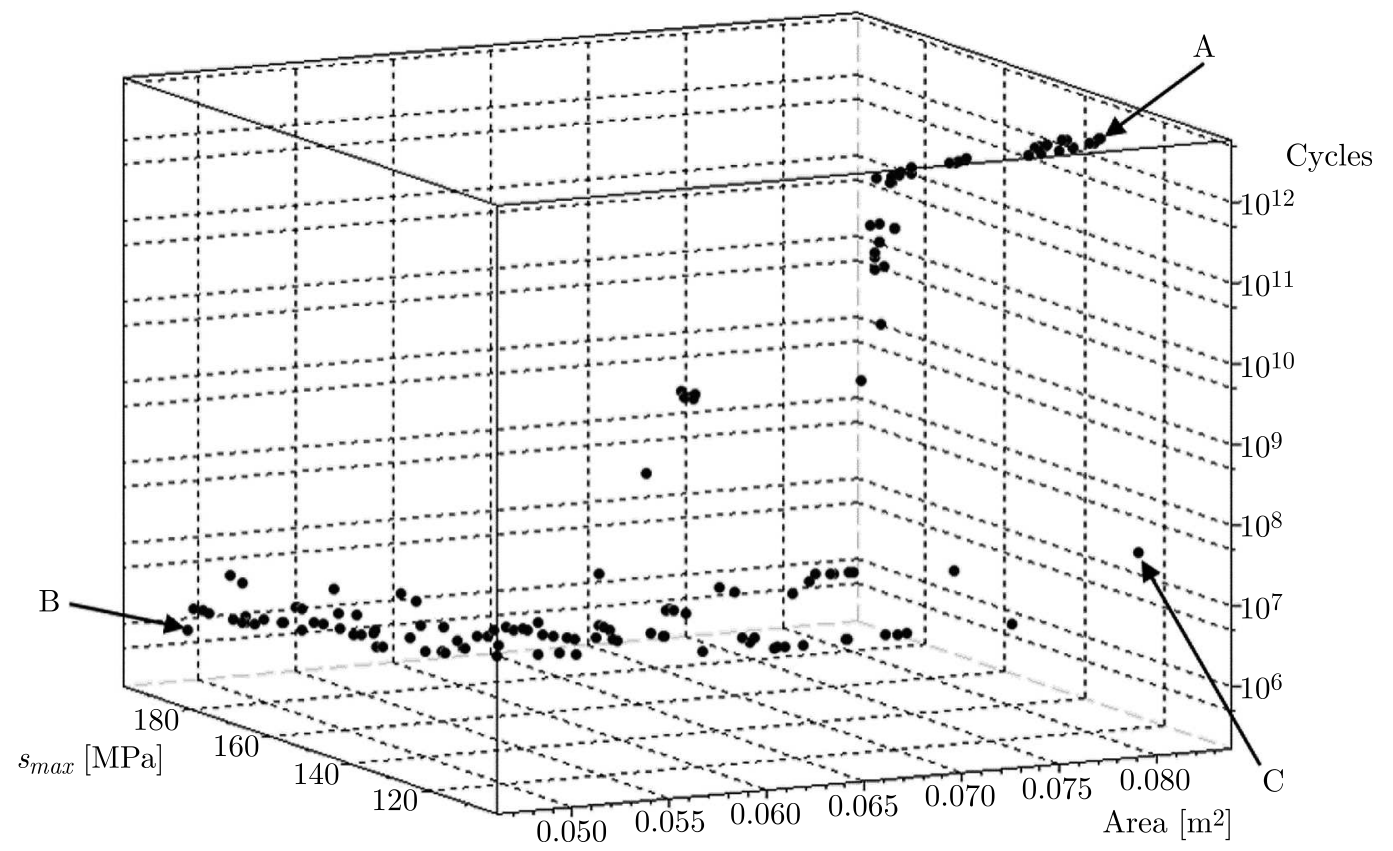

Fig. 6. Pareto front for three-objective optimization

The results of optimization in the form of the final 3D Pareto front and exemplary results for points $A, B$ and $C$ (the best solutions for particular criteria) are presented in Fig. 6 and collected in Table 2.

The figures in Table 2 present optimal shapes (black lines) with the original shape in the background (grey lines). Small circles at optimal shapes denote positions of the maximum values of $\sigma_{e q}$ (and positions of initial crack placing).

It can be observed in Table 2 that different solutions on the Pareto front result with different positions of $\sigma_{\max }$, depending on the optimal shape. As in the previous example, it can be 
Table 2. Optimization results - example 2

\begin{tabular}{|c|c|c|c|}
\hline & $\begin{array}{c}\text { Point } A \\
\max (N(\mathbf{x}))\end{array}$ & $\begin{array}{c}\text { Point } B \\
\min (V(\mathbf{x}))\end{array}$ \\
\hline \hline &
\end{tabular}

observed that the shape of the Pareto front also informs the designer about the sensitivity of particular criteria to the other ones.

\section{Conclusions}

Multiobjective shape optimization of structures with cracks has been performed. To solve the optimization tasks, an in-house multiobjective optimization algorithm MOOPTIM and the dual boundary element method have been combined. Application of NURBS parametric curves allowed arbitrary shape modification with a relatively small number of design variables. The effectiveness of MOOPTIM compared to NSGA-II algorithm has been presented. The use of dBEM instead of FEM resulted in a significant reduction of the calculation time while maintaining high accuracy of the results without the need for remeshing in the case of fracture growth.

Two different cases have been considered: (i) modification of the structure shape to reduce the influence of the identified crack on structure lifetime; (ii) finding the optimal shape of the structural element in order to reduce the risk of arising of the crack and its growth. Different optimization criteria have been considered simultaneously. The optimization results in the form of 2D and 3D Pareto fronts of nondominated solutions have been presented.

The presented numerical examples demonstrate the effectiveness and usefulness of the proposed method of reducing the negative influence of cracks on mechanical structures by means of multiobjective shape optimization. Additional optimization criteria related to other characteristics of cracked structures can be relatively easily introduced and implemented.

\section{Acknowledgement}

The research is partially funded from financial resources from the statutory subsidy of the Faculty of Mechanical Engineering, Silesian University of Technology in 2019.

\section{References}

1. Aliabadi M.H., 2003, Boundary element methods in linear elastic fracture mechanics, Comprehensive Structural Integrity, DOI: 10.1016/B978-0-12-803581-8.00878-X

2. Anderson T.L., 2005, Fracture Mechanics: Fundamentals and Application, CRC Press, Boca Raton, London, New York, Singapore, DOI: 10.1201/9781420058215 
3. Baptista R., Claudio R. A., Reis L., Madeira J. F. A., Guelho I., Freitas M., 2015, Optimization of cruciform specimens for biaxial fatigue loading with direct multi search, Theoretical and Applied Fracture Mechanics, DOI: 10.1016/j.tafmec.2015.06.009

4. Beluch W., 2005, Evolutionary shape optimization in fracture problems, Computer Assisted Mechanics and Engineering Sciences, 12, 2-3, 111-121

5. Beluch W., DŁugosz A., 2016, Multiobjective and multiscale optimization of composite materials by means of evolutionary computations, Journal of Theoretical and Applied Mechanics, DOI: 10.15632/jtam-pl.54.2.397

6. Blandford G.E., Ingraffea A.R., Ligget J.A., 1981, Two-dimensional stress intensity factor computations using the boundary element method, International Journal for Numerical Methods in Engineering, DOI: 10.1002/nme.1620170308

7. Brebiia C.A., Dominiguez J., 1989, Boundary Elements an Introductory Course, Computational Mechanics Publications, Southampton, Boston

8. Brebbia C.A., Walker S., 2016, Boundary Element Techniques in Engineering, Newnes-Butterworths, London-Boston, Sydney, Wellington, Durban, Toronto, DOI: 10.1016/C2013-000814-6

9. Burczyński T., Beluch W., 2001, The identification of cracks using boundary elements and evolutionary algorithms, Engineering Analysis with Boundary Elements, DOI: 10.1016/S09557997(01)00027-3

10. Burczyński T., DŁugosz A., 2012, Multiobjective shape optimization of selected coupled problems by means of evolutionary algorithms, Bulletin of the Polish Academy of Sciences-Technical Sciences, DOI: 10.2478/v10175-012-0028-3

11. Caicedo J., Portela A., 2015, Cracked plate analysis with the dual boundary element method and Williams' eigenexpansion, Engineering Analysis with Boundary Elements, DOI: 10.1016/j.enganabound.2014.11.010

12. Deв K., 1999, Multi-objective genetic algorithms: problem difficulties and construction of test problems, Evolutionary Computation, DOI: 10.1162/evco.1999.7.3.205

13. DeB K., 2001, Multi-Objective Optimization Using Evolutionary Algorithms, John Wiley \& Sons, New York

14. Deb K., Pratap A., Agarwal S., Meyarivan T., 2002, A fast and elitist multi-objective genetic algorithm: NSGA-II, IEEE Transactions on Evolutionary Computation, DOI: 10.1109/4235.996017

15. DŁugosz A., 2013, Multicriteria Optimization in Coupled Field Problems (in Polish), Monograph (497), Silesian University of Technology Publishing House

16. DŁugosz A., 2014, Optimization in multiscale thermoelastic problems, Computer Methods in Materials Science, 14, 1, 86-93

17. Ehrgotт M., 2005, Multicriteria Optimization, Springer-Verlag, Berlin, Heidelberg, DOI: $10.1007 / 3-540-27659-9$

18. Gross D., Seelig T., 2011, Fracture Mechanics: with an Introduction to Micromechanics, Springer-Verlag, Berlin Heidelberg, DOI: 10.1007/978-3-642-19240-1

19. Neimitz A., 1998, Fracture Mechanics (in Polish), Polish Scientific Publishers PWN, Warszawa

20. Perera R., Ruiz A., Manzano C., 2009, Performance assessment of multicriteria damage identification genetic algorithms, Computers and Structures, DOI: 10.1016/j.compstruc.2008.07.003

21. Perera R., Sevillano E., Arteaga A., De Diego A., 2014, Identification of intermediate debonding damage in FRP-plated RC beams based on multi-objective particle swarm optimization without updated baseline model, Composites, Part B: Engineering, DOI: 10.1016/j.compositesb.2014.02.008

22. Piegl L., Tiller W., 1995, The NURBS Book, Springer-Verlag, Berlin, Heidelberg, DOI: 10.1007/978-3-642-97385-7 
23. Portela A., Aliabadi M.H., Rooke D.P., 1992, Dual boundary element method: efficient implementation for cracked problems, International Journal for Numerical Methods in Engineering, DOI: $10.1002 /$ nme.1620330611

24. Shim M.B., SuH M.W., 2010, A study on multiobjective optimization technique for inverse and crack identification problems, Inverse Problems in Engineering, DOI: $10.1080 / 1068276021000008504$

25. Snyder M.D., Cruse T.A., 1975, Boundary integral equation analysis of cracked anisotropic plates, International Journal of Fracture, DOI: 10.1007/BF00038898

26. Sun C.T., Jin Z.-H., 2012, Fracture Mechanics, Academic Press, DOI: 10.1016/C2009-0-63512-1

27. Tada H., Paris P., Irwin G., 2000, The Stress Analysis of Cracks Handbook, 3rd ed., ASME Press, New York, DOI: 10.1115/1.801535

28. Talbi E-G., 2009, Metaheuristics: From Design to Implementation, John Wiley \& Sons, Hoboken, New Jersey, DOI: 10.1002/9780470496916

29. Upadhyaya Y.S., Sridhara B.K., 2011, Fatigue life prediction: a continuum damage mechanics and fracture mechanics approach, Materials and Design, DOI: 10.1016/j.matdes.2011.09.049

30. Zitzler E., Laumanns M., Bleuler S., 2004, A tutorial on evolutionary multiobjective optimization, [In:] Metaheuristics for Multiobjective Optimisation. Lecture Notes in Economics and Mathematical Systems, 535, X. Gandibleux, M. Sevaux, K. Sörensen, V. T'kindt (Eds.), Springer, Berlin, Heidelberg, DOI: 10.1007/978-3-642-17144-4_1 